Conclusion: Our study confirmed that the percentage of patients showing low adherence to therapy is relevant. Moreover, the association of lower adherence to treatments with higher values of the subjective clinimetric indexes suggests to pay attention to the apparent ineffectiveness or loss of efficacy of therapy. References:

[1] Morisky DE et al. J Clin Hypertens 2008; 10:348-354.

Disclosure of Interests: None declared

DOI: 10.1136/annrheumdis-2020-eular.6069

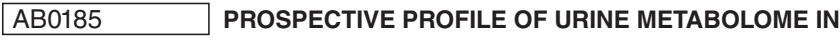 RHEUMATOID ARTHRITIS}

M. De Oliveira ${ }^{1}$, P. V. Alabarse ${ }^{2}$, M. Farinon ${ }^{1}$, R. Cavalheiro Do Espírito Santo ${ }^{1}$, R. Xavier ${ }^{1}$ on behalf of Department of Rheumatology - Clinicas Hospital of Porto Alegre, Brazil - Federal University of Rio Grande do Sul. ${ }^{1}$ Federal University of Rio Grande do Sul, Porto Alegre, Brazil; ${ }^{2}$ University of San Diego, San Diego, United States of America

Background: Rheumatoid arthritis $(\mathrm{RA})$ is a chronic inflammatory disease characterized by increased mortality and associated with metabolic disorders. Since the metabolomic profile is known to vary in response to different inflammatory conditions, metabolome analysis could substantially improve diagnosis and prognosis of RA.

Objectives: To analyze the urine metabolome profile in RA patients and correlate it with disease activity changes over 12 months

Methods: Seventy-nine RA patients, according to ACR/EULAR 2010 classification criteria, between 40 and 70 years old, were recruited and followed for 12 months. Metabolome analysis was performed by Nuclear Magnetic Resonance spectroscopy (NMR), resulting in the identification of 93 metabolites in urine collected at the baseline and after 12 months. Frequency analysis, Pearson Correlation and Multivariate data analysis with orthogonal projections to latent structures (OPLS) method were performed and a statistical significance was considered as $p<0.05$. Results: The study population was characterized by the majority of women $(86.7 \%)$, mean age of 56 years old, around $80 \%$ with positive anti-CCP or Rheumatoid Factor. During the one year of follow-up, there was no substantial variation in the DAS28 measurement (baseline: 3.8, after 12 months: 4.0). There was no significant correlation between the metabolome pattern and DAS28 score $(p>0.05)$ over time. However, multivariate analysis (OPLS-DA) demonstrated an adequate differentiation of the population with 0.92 of accuracy (Q2: 0.72 and R2: 0.89).There was a significant increase of L-cysteine, choline, L-Phenylalanin, creatine, L-histidine, oxalacetic acid and xanthine, and a decrease of L-threonine, taurine, butyric and gluconic acid $(p<0.05)$ during the follow-up, metabolites that are involved in the skeletal muscle metabolism.

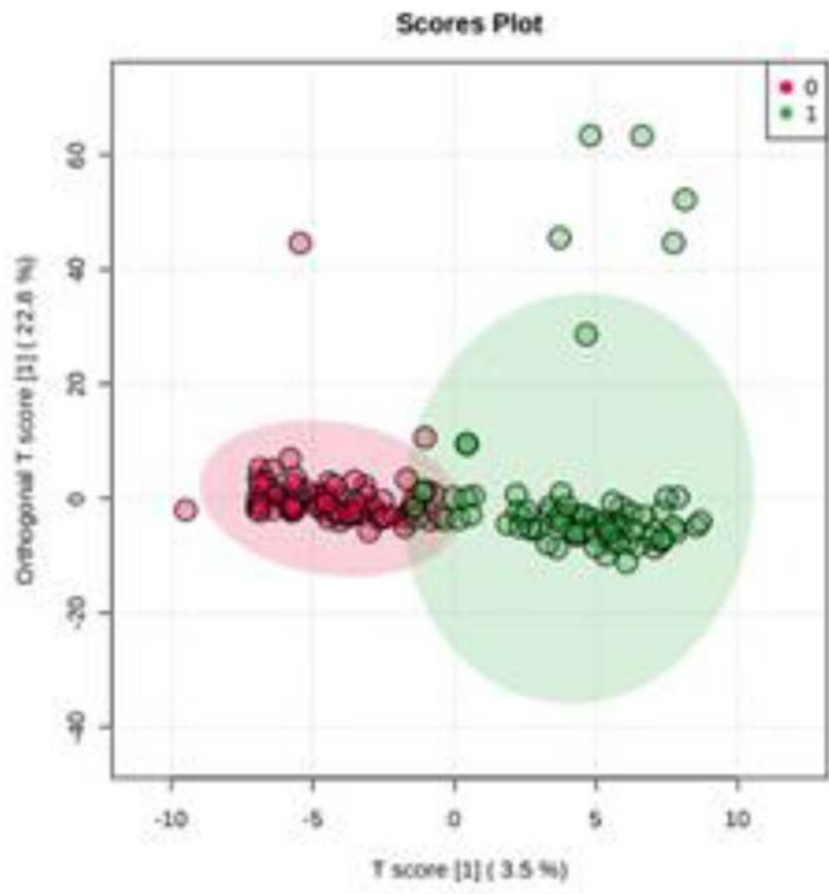

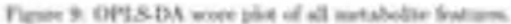

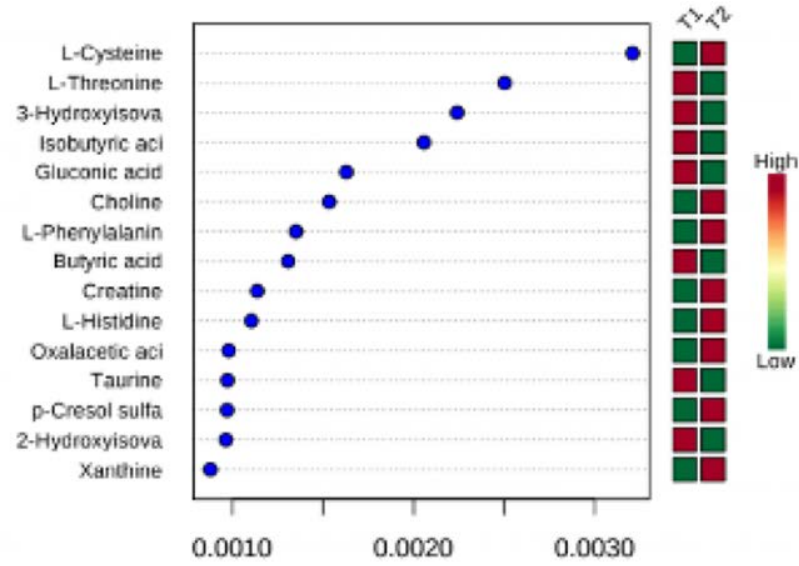

MeanDecreaseAccuracy

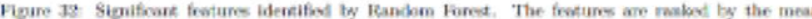
drecese in daesifratice axuracy wbos they are perminied.

Conclusion: The observed biomarkers indicate, as expected, that the RA metabolic profile is associated with inflammation injury and skeletal muscle amino acid metabolism. Correlations with disease activity changes was compromised by the stable disease status during the 12 months. More studies evaluating correlations with skeletal muscle function and mass are underway.

Acknowledgments: Disclosure of interest: Marianne Oliveira: None declared, Rafaela Santo: None declared, Mirian Farinon: None declared, Ricardo Xavier Consultant of: Abbvie, Pfizer, Novartis, Janssen, Lilly, Roche

Disclosure of Interests: Marianne de Oliveira: None declared, Paulo Vinicius Alabarse: None declared, Mirian Farinon: None declared, Rafaela Cavalheiro do Espírito Santo: None declared, Ricardo Xavier Consultant of: AbbVie, Pfizer, Novartis, Janssen, Eli Lilly, Roche

DOI: 10.1136/annrheumdis-2020-eular.4481

\section{AB0186 NO PREDICTIVE VALUE OF ADALIMUMAB SERUM LEVELS AND ANTI-ADALIMUMAB ANTIBODIES AT TIME OF ADALIMUMAB FAILURE FOR PREDICTION OF RESPONSE TO THE NEXT BDMARD}

N. Den Broeder ${ }^{1}$, E. Ulijn ${ }^{1}$, M. Wientjes ${ }^{1}$, N. Van Herwaarden ${ }^{1,2}$, I. Meek ${ }^{2}$, L. Tweehuysen ${ }^{1}$, A. Van der Maas ${ }^{1}$, B. Van den Bemt ${ }^{3,4}$, A. Den Broeder ${ }^{1}$. ${ }^{1}$ Sint Maartenskliniek, Rheumatology, Nijmegen, Netherlands; ${ }^{2}$ Radboudumc, Rheumatology, Nijmegen, Netherlands; ${ }^{3}$ Sint Maartenskliniek, Pharmacy, Nijmegen, Netherlands; ${ }^{4}$ Radboudumc, Pharmacy, Nijmegen, Netherlands

Background: After adalimumab treatment failure, TNFi and non-TNFi bDMARDs are equally viable as subsequent treatment in RA. However, preliminary data suggest that anti-drug antibodies (ADA) and adalimumab serum levels (ADL) predict response to a subsequent TNFi [1].

Objectives: To assess the association of presence of ADA and/or low ADL with response to a subsequent TNFi bDMARD or non-TNFi bDMARD.

Methods: A retrospective cohort study to assess the predictive value of ADA and ADL for response to a subsequent TNFi or non-TNFi bDMARD in RA patients. All RA patients who received adalimumab (standard dose, $\geq 3$ months) and subsequently switched to another TNFi or a non-TNFi (rituximab, tocilizumab, abatacept) in the Sint Maartenskliniek or Radboud University Medical Centre between January 2012 and January 2018 were considered for inclusion in the current study. Further inclusion criteria were the availability of (random timed) serum samples between $\geq 8$ weeks after start, and $\leq 2$ weeks (for ADL) or $\leq 12$ weeks (for ADA) after discontinuation of adalimumab, and clinical outcome measurements (DAS28-CRP/BSE) between 3-6 months after treatment switch Serum samples were derived from a period of biobanking at every visit of RA patients and an observational cohort study including consecutive bDMARD starters.

The primary outcome of this study was the association between ADL or ADA and EULAR good response (DAS28-CRP/ESR based) to the subsequent bDMARD. When DAS28-based response was unreliable due to glucocorticoid use, or low baseline DAS28 (if switching due to adverse effects), judgement of the rheumatologist was used.

A drug-tolerant competitive enzyme-linked immunosorbent assay (Sanquin, the Netherlands) was used to quantify ADA, and thereafter, ADL was determined via an ELISA. Reference values were $\geq 5 \mu \mathrm{g} / \mathrm{ml}$ for $\mathrm{ADL}$ and $<12 \mathrm{AU} / \mathrm{ml}$ for ADA [2,3] Treatment was blinded for ADL and ADA levels. 
Prediction of response were assessed using the area under the receiver-operator characteristic (AUROC) and sensitivity/specificity. Sub-analyses were performed for primary and secondary non-responders. Correlations between ADL and ADA presence and clinical variables were also cross-sectionally explored.

Results: 137 patients were included, 47 of whom switched to a second TNFi and 90 to a non-TNFi. Sensitivity and specificity of the proposed ADA and ADL reference values were low (table 1). The AUROC did not differ appreciably or significantly from 0.5 . Results were similar for both primary and secondary nonresponders to adalimumab.

Table 1. predictive values of ADA and ADL for response to a subsequent bDMARD in TNFi and non-TNFi switchers.

\begin{tabular}{lcccc}
\hline & sensitivity (\%) & specificity (\%) & AUC & Cl \\
\hline & TNFi switchers & & & \\
\hline ADA presence $(>12 \mathrm{AU} / \mathrm{mL})$ & 18 & 75 & 0.46 & $0.32-0.59$ \\
low ADL $(<5 \mathrm{mg} / \mathrm{L})$ & 32 & 69 & 0.50 & $0.29-0.71$ \\
& non-TNFi switchers & & & \\
ADA presence $(>12 \mathrm{AU} / \mathrm{mL})$ & 33 & 70 & 0.52 & $0.42-0.63$ \\
low ADL $(<5 \mathrm{mg} / \mathrm{L})$ & 50 & 52 & 0.50 & $0.34-0.65$
\end{tabular}

Higher ADL (Spearman's $\rho=-0.68, p=0.00)$ but not ADA $(\rho=0.23, p=0.28)$ presence was associated with a lower DAS28 at the time of switching to a subsequent bDMARD, but not with follow-up DAS28 after starting the subsequent bDMARD $(\rho=-0.29, p=0.17$, and $\rho=0.10, p=0.65$, respectively). In addition, higher ADL were associated with lower baseline CRP $(\rho=-0.67, p=0.00)$ and ESR $(\rho=-0.546, p=0.006)$ and higher ADA correlated with higher baseline ESR $(\rho=0.49, p=0.01)$.

Conclusion: No predictive value for response to a second TNFi or non-TNFi was found for either ADA or random timed ADL. Limitations of this study are the retrospective design and random timed serum sampling. An ongoing randomized blinded test-treatment trial will provide more definitive answers [4].

References:

[1] Van Herwaarden et al. Expert Opin Drug Metab Toxicol. 2017;13:843-57.

[2] Pouw et al. Ann Rheum Dis. 2015 Mar;74(3):513-8.

[3] Bartelds et al. Ann Rheum Dis. 2007 Jul;66(7):921-6.

[4] ADDORA-SWITCH study, www.trialregister.nl, no NL 8210

Disclosure of Interests: Nathan den Broeder: None declared, Evy Ulijn: None declared, Maike Wientjes: None declared, Noortje van Herwaarden: None declared, Inger Meek: None declared, Lieke Tweehuysen: None declared, Aatke van der Maas: None declared, Bart van den Bemt Grant/research support from: UCB, Pfizer and Abbvie, Consultant of: Delivered consultancy work for UCB, Novartis and Pfizer, Speakers bureau: Pfizer, AbbVie, UCB, Biogen and Sandoz., Alfons den Broeder: None declared

DOI: 10.1136/annrheumdis-2020-eular.1386

\section{AB0187 1 ASSESSMENT OF ADHERENCE TO TREATMENT OF PATIENTS WITH RHEUMATOID ARTHRITIS (RA)}

E. Egorova ${ }^{1}$, N. Nikitina ${ }^{1}$, A. Rebrov ${ }^{1} .{ }^{1}$ Saratov State Medical University named V. I. Razumovsky, Ministry of Health of Russia, Saratov, Russian Federation

Background: Rheumatoid arthritis (RA) is the most common chronic immune inflammatory disease. The effectiveness of RA therapy largely depends on adherence to treatment. Non-compliance with the recommendations of the doctor leads to increased disease activity, a greater risk of complications and the increase in the cost of treatment.

Objectives: To determine predictors of adherence to treatment of patients with RA. Methods: The study included 82 women with reliable RA according to the criteria of ACR1987 and / or EULAR / ACR2010 (mean age 53.3 \pm 10.2 years, the age at the onset of the disease is 42.4 [36;51] years, mean duration of RA - $10.8[6 ; 14]$ years, DAS28 - 5.03 [4.3;5.8]). Treatment adherence was assessed according to the questionnaire "Quantitative Evaluation of Adherence to Treatment (KOP - 25)" [1]. The following indicators were calculated: adherence to drug therapy, adherence to medical support, adherence to lifestyle modification and their integral index. For all indicators, the level of values in the range up to $50 \%$ is interpreted as "low" («non-adherence to treatment»), from 51 to $75 \%$ - as "medium", more than $75 \%$ - as "high"(«adherence to treatment»). The functional ability of patients was assessed by the Health Assessment Questionnaire (HAQ). The severity of pain was determined by VAS. Statistical processing was performing using the program STATISTICA 10.0.

Results: Adherence to drug therapy in women with RA was determined: low adherence in 32 (39\%) patients, average in 34 (41.5\%) patients and high in 16 (19.5\%) patients; adherence to medical support: low in $26(31.7 \%)$ patients, average in $40(48.8 \%)$ patients, and high in $16(19.5 \%)$ patients; adherence to lifestyle modification: low in 55 (67\%) patients, average in 25 $(30.5 \%)$ patients and high in $2(2.5 \%)$ patients. According to the integral indicator of adherence to treatment, 34 (41.5\%) patients were not adherent to treatment, average adherence was recorded in $42(51.2 \%)$ patients, and high in $6(7.3 \%)$ patients.

The HAQ functional impairment was absent in 7 (8.5\%) patients, minimal impairment occurred in $26(31.7 \%)$, moderate - in $40(48.8 \%)$ and severe - in $9(11 \%)$ patients.

Severe pain in the VAS was noted by 29 (35.4\%) patients, moderate - 39 (47.6\%) in $14(17 \%)$ patients the pain syndrome was weakly expressed.

The relationships of adherence to treatment was established with age $(r=-0.29$ $p<0.05)$, age at the onset of the disease $(r=-0.28, p<0.05), H A Q$ index $(r=$ $-0.27, p<0.05)$, the number of swollen joints $(r=-0.3, p<0.05)$.

Patients under age of 39 years were the most adherence to drug therapy.

In patients with medium and high adhering to treatment, the severity of pain according to VAS was significantly lower than in non-adherent patients (50.6 [34;66] and 60.4 [46;73], respectively, $p=0.04)$, In patients with treatment adherence, activity was significantly lower than in non-treatment adherents (DAS 28 $(4.7$ [3.5;5.4] and $5.3[4.7 ; 5.9]$, respectively, $p=0.04)$

Conclusion: Low treatment adherence has $41,5 \%$ of RA patients. Predictors of adherence to treatment are the young age patients, the onset of the disease before age of 39 years. Non-treatment patients with RA have a higher activity of RA according to DAS28, pain intensity according to VAS, the worst functional status. To increase the effectiveness of treatment, constant interaction between the patient and the physician is necessary, explaining to patients the consequences of non-compliance with recommendations.

References:

[1] Nikolaev N. A., Skirdenko Yu. P. Russian universal questionnaire for the quantitative evaluation of adherence to treatment (KOP - 25). Clinical pharmacology and therapy. 2018;27(1): 74-78.

Disclosure of Interests: None declared

DOI: 10.1136/annrheumdis-2020-eular.1780

\section{AB0188 COMPARISON OF COMPOSITE INDICES FOR DETECTING REMISSION ON ULTRASOUND}

R. Fakhfakh ${ }^{1}$, N. El Amri ${ }^{1}$, K. Baccouche ${ }^{1}$, H. Zeglaoui ${ }^{1}$, E. Bouajina ${ }^{1} .{ }^{1}$ Farhat Hached Hospital, Rheumatology, Sousse, Tunisia

Background: Several studies have shown the greater sensitivity of ultrasound (US) to detect B-mode synovitis and synovial Doppler activity in a high percentage of rheumatoid Arthritis (RA) patients in clinical remission, assessed by different composite indices.

Objectives: The aim of the study was to compare the accuracy of composite indices to detect remission in ultrasound B-mode and power Doppler (PD) in RA patients that are in remission according to the DAS28 ESR

Methods: Cross-sectional study including patients with RA in clinical remission defined by: DAS28ESR $\leq 2.6$, without disease flare or changes in therapy in the previous 6 months. Each patient underwent B-mode and PD assessments of 36 joints and 20 tendons in the Rheumatology Department over a period of 6 month. B-mode and PD signal for synovitis and tenosynovitis were defined according to the Outcome Measures in Rheumatology Clinical Trials (OMERACT). A global score for B-mode and a global score for PD signal were calculated for each patient. The DAS28, CDAI, SDAI and the Boolean 2010 ACR/EULAR remission criteria were compared.

Results: Thirty two patients were enrolled, the mean age was $53.7 \pm 13.4$ and the sex ratio $\mathrm{M} / \mathrm{F}$ was 0.3 . The mean disease duration was 15.0 years \pm 8.8 According to the SDAI, $68.8 \%$ of patients were in remission. These were lower for the CDAI (62.5\%) and the Boolean criteria (23.3\%). Synovial hypertrophy and tenosynovitis in B mode was detected in $100 \%$ with the Boolean remission criteria, in $93.8 \%$ with a DAS28, in $90.9 \%$ with a SDAI $\leq 3.3$ and in $90 \%$ with a CDAI $\leq 2.8$ ( $p>0.05$ ). The PD signal was detected in $62.5 \%$ with a DAS28, in $59.1 \%$ with a SDAI $\leq 3.3$, in $57.1 \%$ with the Boolean remission criteria and in $55.1 \%$ with a CDAI $\leq 2.8$ ( $p>0.05)$. The mean B-mode global score was higher for the DAS28 ESR $(8.2 \pm 6)$ and lower for the Boolean remission criteria (6.2 \pm 5.4$)$. For a CDAI $\leq 2.8$, the mean global score for $\mathrm{B}$-mode was $7.6 \pm 5.9$ and for a $\mathrm{SDAI} \leq 3.3$, it was $7.4 \pm 5.7$. The median PD global score was similar for the DAS28, SDAI $\leq 3.3$ and Boolean remission criteria (1[0-12]). It was higher for a CDAI $\leq 2.8$ (1.5 [0-12]) The global score for PD signal was correlated with DAS28 ESR ( $r: 0.42 \mathrm{p}: 0.02$ ). There were no significant correlations between the other indices and the mode $B$ and PD global scores.

Conclusion: The CDAI least detected subclinical synovitis and tenosynovitis in B mode and in power Doppler signal but it showed higher scores of power Doppler.

References:

[1] Merve Ozata Olmez, Esen Kasapoglu Gunal, Sibel Bakirci Ureyen and al. Comparison of composite indices with global synovitis score on ultrasound for detecting remission. Clinical Rheumatology 2017. doi.org/10.1007/ s10067-017-3925-x 